\title{
TITLE:
}

\section{Visualization of Notch signaling oscillation in cells and tissues.}

\section{$\operatorname{AUTHOR}(\mathrm{S})$ :}

Shimojo, Hiromi; Harima, Yukiko; Kageyama, Ryoichiro

\section{CITATION:}

Shimojo, Hiromi ... [et al]. Visualization of Notch signaling oscillation in cells and tissues.. Methods in molecular biology 2014, 1187: 169-179

\section{ISSUE DATE:}

2014-06-26

URL:

http://hdl.handle.net/2433/198460

\section{RIGHT:}

The final publication is available at Springer via http://dx.doi.org/10.1007/978-1-49391139-4_13; 許諾条件により本文ファイルは2015-06-26に公開.; This is not the published version. Please cite only the published version.; この論文は出版社版でありません。引用 の際には出版社版をご確認ご利用ください。 


\section{Visualization of Notch signaling oscillation in cells and tissues}

Hiromi Shimojo $^{1,2}$, Yukiko Harima ${ }^{1,3}$, and Ryoichiro Kageyama ${ }^{1,2,3^{*}}$

${ }^{1}$ Institute for Virus Research, Kyoto University, Shogoin-Kawahara, Sakyo-ku, Kyoto 606-8507, Japan

${ }^{2}$ World Premier International Research Initiative-Institute for Integrated Cell-Material Sciences (WPI-iCeMS), Kyoto University, Kyoto 606-8501, Japan

${ }^{3}$ Japan Science and Technology Agency, Core Research for Evolutional Science and Technology (CREST), 4-1-8 Honcho, Kawaguchi, Saitama, 332-0012, Japan

* Correspondence to: Ryoichiro Kageyama, Institute for Virus Research, Kyoto University, Shogoin-Kawahara, Sakyo-ku, Kyoto 606-8507, Japan. E-mail: rkageyam@virus.kyoto-u.ac.jp Running title: Visualization of Notch signaling oscillation 


\section{Summary}

The Notch signaling effectors Hes 1 and Hes7 exhibit oscillatory expression with a period of about 2-3 hours during embryogenesis. Hes1 oscillation is important for proliferation and differentiation of neural stem cells, whereas Hes7 oscillation regulates periodic formation of somites. Continuous expression of Hes1 and Hes7 inhibits these developmental processes. Thus, expression dynamics are very important for gene functions, but it is difficult to distinguish between oscillatory and persistent expression by conventional methods such as in situ hybridization and immunostaining. Here, we describe time-lapse imaging methods using destabilized luciferase reporters and a highly sensitive cooled charge-coupled device camera, which can monitor dynamic gene expression. Furthermore, the expression of two genes can be examined simultaneously by a dual reporter system using two-color luciferase reporters. Timelapse imaging analyses reveal how dynamically gene expression changes in many biological events.

Keywords: Hes1, Hes7, Oscillatory expression; Luciferase, Bioluminescence; CCD, Neural stem cell; Presomitic mesoderm; Segmentation clock 


\section{Introduction}

The expression of the mouse Notch effector Hes1 oscillates with a period of about 2-3 hours in many cell types, including neural stem cells (1-4). Activation of Notch signaling releases the Notch intracellular domain (NICD), which is then transferred to the nucleus, inducing Hes1 expression (5). Hes1, a transcriptional repressor, directly binds to the Hes1 promoter and represses its own expression (1,6). When the Hes1 promoter is repressed, both Hes 1 mRNA and Hes 1 protein disappear rapidly, because they are extremely unstable, and this allows the next round of expression. Thus, Hes 1 expression autonomously oscillates by a negative feedback. Hes1 oscillation is important for proliferation and differentiation of neural stem cells, because steady expression of Hes1 inhibits both processes (7). The expression of another mouse Notch effector, Hes7, also oscillates with a period of about 2 hours in the presomitic mesoderm (PSM), which is located in the caudal end of embryos. Hes7 oscillation leads to periodic segmentation of the rostral parts of the PSM, thereby forming a bilateral pair of somites every two hours. Steady expression of Hes7 leads to severe fusion of somites, indicating that oscillatory expression is required for periodic somite formation $(\mathbf{8 , 9})$.

It is difficult to analyze oscillatory expression by conventional methods such as in situ hybridization and immunostaining, which cannot adequately capture the dynamics of gene expression. Time-lapse imaging of reporter expression is a powerful approach to analyze dynamic gene expression. Because the period of Hes1 and Hes7 oscillations is short (2-3 hours), the response of reporter expression must be rapid. To this end, we use luciferase as a reporter rather than fluorescent proteins such as GFP, because luciferase immediately generates luminescence in the presence of its substrate, luciferin, whereas fluorescent proteins require at 
least a few hours to properly fold in order to emit fluorescence. However, because wild-type luciferase is too stable to monitor oscillatory expression, a form destabilized by ubiquitination is used (ubiquitinated luciferase, Ub-Luc) (2). A highly sensitive cooled charge-coupled device (CCD) camera successfully captures the bioluminescence from cells and tissues carrying the Hes1 or Hes7 promoter-driven Ub-Luc reporters (2-4,10-12). In this chapter, we describe detailed methods to monitor oscillatory gene expression in cultured PSM tissues and neural stem cells. We also describe a dual color luciferase reporter method, which is able to monitor the expression of two genes in the same cells.

\section{Materials}

\subsection{Ub-Luc reporter construction}

We fused the coding region of one copy of a mutant ubiquitin (G76V), which resists cleavage by ubiquitin hydrolases, to firefly luciferase cDNA in frame at the 5' terminus to generate Ub-Luc (13). To make the pHes1-Ub-Luc reporter, the Hes1 promoter and 5'UTR region $(-2567$ to +223$)$, the Ub-Luc cDNA, the Hes1 3'UTR (+2090 to +2453$)$ and the downstream region $(+2454$ to +2626) were ligated in this order (Fig. 1A) (2). To make the pHes7-Ub-Luc reporter, the Hes7 promoter and 5'UTR region (5393 bp upstream fragment from the first codon), the Ub-Luc cDNA, the Hes 7 3'UTR and the region 76 bp downstream of the putative polyadenylation signal were ligated in this order (10). Transgenic mice carrying pHes1-Ub-Luc or pHes7-Ub-Luc were generated using these constructs.

\subsection{Bioluminescence imaging system}


For setting up the bioluminescence imaging system, prepare a dark room and keep the temperature and humidity low (Temperature is kept at $20-26^{\circ} \mathrm{C}$, and humidity is less than $\sim 50 \%$ ) for maintenance of the instruments. We use the following two systems, which show similar sensitivity.

1. Inverted microscope (Olympus IX81) carrying cooled CCD camera (Princeton Instruments, VersArray $1 \mathrm{~kb})(2)$.

2. Inverted microscope (Olympus IX81) carrying cooled CCD camera (Andor Technology iKon-M 934).

We use Image-Pro Plus (Media Cybemetics) software to acquire the images.

\subsection{Explant culture of the caudal part of mouse embryos}

1. DMEM supplemented with $10 \%$ fetal bovine serum and $1 \%$ penicillin/streptomycin.

2. Tissue culture dish $(6 \mathrm{~cm})$.

3. Surgical scissors, fine.

4. Two pairs of forceps, fine.

5. 35-mm glass-based dishes ( $\phi 12-m m$ glass, e.g. IWAKI \#3911-035).

6. D-luciferin sodium salt (e.g. Nacalai Tesque \#01493-85).

\subsection{Neural stem cell dissociation culture}

1. Neurosphere culture medium: DMEM/F12 supplemented with $100 \mu \mathrm{g} / \mathrm{ml}$ transferrin, $25 \mu \mathrm{g} / \mathrm{ml}$ insulin, 20nM progesterone, 30nM sodium selenite, $60 \mu \mathrm{M}$ putrescine, $20 \mathrm{ng} / \mathrm{ml}$ EGF, 20ng/ml bFGF and 1\% penicillin/streptomycin (see Note 1) (3).

2. $0.25 \%$ Trypsin/EDTA (e.g. GIBCO). 
3. $2000 \mathrm{U} / \mathrm{ml}$ DNaseI.

4. $2 \%$ Trypsin inhibitor.

5. N2 and B27 medium: DMEM/F12 supplemented with 1x B27 (GIBCO \#12587-010), 1x N2 (GIBCO \#17502-048), 1 $\mathrm{mM} \mathrm{N-acetyl-cysteine,} \mathrm{10ng/ml} \mathrm{bFGF} \mathrm{and} \mathrm{penicillin/streptomycin}$ (see Note 1) (14).

6. $7 \mathrm{U} / \mathrm{ml}$ papain solution (see Note 2).

7. $0.06 \%$ DNase solution (see Note 2 ).

8. 1M N-acetyl-cysteine (NAC) (Sigma).

9. $35 \mathrm{~mm}$ glass-based dishes coated with $40 \mu \mathrm{g} / \mathrm{ml}$ poly-L-lysine (see Note 3).

10. D-luciferin sodium salt (see Note 4).

\subsection{Slice culture of the dorsal telencephalon of mouse embryos}

1. Basal medium: DMEM/F12 supplemented with penicillin/streptomycin.

2. Enriched slice culture medium: DMEM/F12 supplemented with $100 \mu \mathrm{g} / \mathrm{ml}$ transferrin, $25 \mu \mathrm{g} / \mathrm{ml}$ insulin, $20 \mathrm{nM}$ progesterone, $30 \mathrm{nM}$ sodium selenite, $60 \mu \mathrm{M}$ putrescine, $20 \mathrm{ng} / \mathrm{ml} \mathrm{EGF}$, $20 \mathrm{ng} / \mathrm{ml} \mathrm{bFGF,} \mathrm{5 \%} \mathrm{horse} \mathrm{serum,} 5 \%$ fetal bovine serum and penicillin/streptomycin $(3,15)$.

3. $35 \mathrm{~mm}$ glass-based dishes.

4. Millicell cell culture inserts (Millipore \#PICM 01250) (see Note 5).

5. D-luciferin sodium salt.

\subsection{Dual reporter system of red-/green-color luciferase}

1. Reporters: Destabilized multicolor luciferase was constructed as follows. The coding region of a mutant ubiquitin (G76V) was fused to red-color luciferase (SLR) or green-color 
luciferase (ELuc) cDNA in-frame at the 5' terminus (Fig. 1B, see Note 6) (11). These

luciferases utilize the same substrate, D-luciferin, but generate different color luminescence.

2. Optimized emission filters (see Note 7).

3. D-luciferin sodium salt.

\section{Methods}

\subsection{Bioluminescence imaging of Hes1 and Hes7 expression in PSM-containing explant culture}

1. Sacrifice a pregnant female mouse by cervical dislocation. We usually use embryos at day 10.5 (E10.5) for the analysis of PSM tissues.

2. Lay the pregnant mouse on a bench, and soak the abdominal region with $70 \%$ ethanol to prevent contamination.

3. Pinch the abdominal skin and make a small lateral incision at the midline with surgical scissors.

4. Hold the skin and pull toward the head and tail until abdomen is completely exposed.

5. Cut the body wall along the midline.

6. Grasp the cervix and pull up the uterus with forceps. Then, cut the cervix with scissors.

7. Put the uterus in a tissue culture dish containing pre-warmed PBS and wash blood away.

8. Put the uterus in a new dish containing pre-warmed medium. This medium contains DMEM supplemented $10 \%$ FBS and $1 \%$ penicillin/streptomycin.

9. Pull out all embryos from the uterus, and put them in a new dish containing pre-warmed medium under a dissecting microscope (see Note 8).

10. Remove the muscle layer and peel the yolk sac carefully. 
11. Cut the tails of all embryos. The cutting point is just rostral to the last 2 segmented somites (Fig. 2, see Note 9).

12. Pick up these tips of tails with pipette and transfer into $35 \mathrm{~mm}$ glass-based dishes $(\phi 12-\mathrm{mm}$ glass) containing $350 \mu \mathrm{l}$ drop of pre-warmed culture media (DMEM supplemented with 10\% FBS, 1\% penicillin/streptomycin and 1mM luciferin) (see Note 10).

13. Put this glass-based dish on the stage of inverted microscope (Olympus IX81) and incubate in $5 \% \mathrm{CO}_{2}$ and $85 \% \mathrm{O}_{2}$ at $37^{\circ} \mathrm{C}$ (see Note 11 ).

14. To genotype the samples, collect the tips of tails in the center of the dish and observe the luminescence using an Olympus x4 UPlanSApo (or similar) objective lens (numerical aperture (NA): 0.16 ) at $4.5 \mathrm{~min}$ exposure.

15. Select a transgene-positive and straight shaped tail (see Note 12).

16. Put the selected tail on the center of dish (Fig. 2) and set an Olympus x20 UPlanApo objective lens (NA: 0.8) transmitted directly to a cooled CCD camera. The signal-to-noise ratio can be increased by $4 \times 4$ binning. For this particular experiment, exposure time was set at $9.5 \mathrm{~min}$. Record the images in 16 bit using Image-Pro Plus (Media Cybemetics) (see Note 13).

17. Analyze the image with ImageJ software (see Note 14).

\subsection{Bioluminescence imaging of Hes1 expression in neural stem cell dissociation cultures}

\subsubsection{Isolation of cells using Trypsin/EDTA}

1. Dissect out the uterus from pregnant mice carrying embryos at E11.5-14.5, as described above (steps 1-6 of 3.1.).

2. Wash the uterus in ice-cold PBS. 
3. Dissect out embryos from the uterus.

4. Transfer the embryos into ice-cold DMEM/F12 medium.

5. Excise the telencephalon.

6. Transfer the telencephalon into ice-cold neurosphere culture medium.

7. Remove mesenchyme, meninges and ganglionic eminence, and take the cortex.

8. Transfer the cortex into a disposable $15 \mathrm{ml}$ tube.

From this step onwards, carry out on a clean bench to avoid contamination.

9. Discard the supernatant and extra medium.

10. Add $1 \mathrm{ml} 0.25 \%$ Trypsin/EDTA and $200 \mu \mathrm{l}$ DNaseI $(2000 \mathrm{U} / \mathrm{ml})$ to digest DNAs released from dead cells.

11. Pipet gently with $1 \mathrm{ml}$ tip 5 to 10 times.

12. Incubate at $37^{\circ} \mathrm{C}$ for $5 \mathrm{~min}$.

13. Pipet gently with $1 \mathrm{ml}$ tip 10 to 20 times.

14. Incubate at $37^{\circ} \mathrm{C}$ for $5 \mathrm{~min}$ again.

15. Pipet gently with $1 \mathrm{ml}$ tip 10 to 20 times.

16. Add $100 \mu 12 \%$ Trypsin inhibitor to stop the enzyme reaction.

17. Pipet gently with $1 \mathrm{ml}$ tip 10 times.

18. Add 5ml DMEM and pipet 10 times.

19. Centrifuge the mixture at $1000 \mathrm{rpm}$ for $5 \mathrm{~min}$ at room temperature.

20. Discard the supernatant and suspend the cell pellet in neurosphere culture medium containing $1 \mathrm{mM}$ D-luciferin (see Note 15).

21. Plate the cells in PLL-coated glass-based dishes (see Note 15).

22. Place the dish on the stage of inverted microscope of the imaging system. 
23. Cultured cells are maintained at $37^{\circ} \mathrm{C}$ in $5 \% \mathrm{CO}_{2}$.

24. Bioluminescence from the reporter is monitored by an Olympus x40 UPlanApo objective and is transmitted directly to CCD camera (Fig. 3).

\subsubsection{Isolation of cells using papain solution}

This treatment is milder than Trypsin/EDTA and is often used for younger embryos.

1. Dissect out the uterus from pregnant mice carrying embryos at E11.5-14.5, as described above (steps 1-6 of 3.1.).

2. Wash the uterus in ice-cold PBS.

3. Dissect out embryos from the uterus.

4. Transfer the embryos into ice-cold DMEM/F12 medium.

5. Excise the telencephalon.

6. Transfer the telencephalon into ice-cold N2 and B27 medium to maintain the undifferentiated state of neural stem cells.

7. Remove mesenchyme, meninges and ganglionic eminence, and take the cortex.

8. Transfer the cortex into disposable $15 \mathrm{ml}$ tube.

From this step onwards, carry out on a clean bench.

9. Discard the supernatant and extra medium.

10. Add $1 \mathrm{ml} 7 \mathrm{U} / \mathrm{ml}$ papain solution, $100 \mu 10.06 \%$ DNase solution and $1 \mu 11 \mathrm{mM} \mathrm{NAC} \mathrm{(dissolved}$ in distilled water), which restores the intracellular antioxidant glutathione.

11. Incubate and shake the mixture at room temperature $\left(22-24^{\circ} \mathrm{C}\right)$ for $30 \mathrm{~min}$.

12. Pipet gently with $1 \mathrm{ml}$ tip 10 times.

13. Centrifuge the mixture at $1200 \mathrm{rpm}$ for $5 \mathrm{~min}$ at room temperature. 
14. Discard the supernatant. Add $1 \mathrm{ml}$ DMEM/F12, and suspend the pellet.

15. Add 2ml DMEM/F12 and mix.

16. Repeat the wash steps (steps 13-15) 3 times.

17. Discard the supernatant and suspend the pellet in N2 and B27 medium containing 1mM Dluciferin.

18. Plate the cells in PLL-coated glass-based dishes (see Note 15).

19. Proceed to bioluminescence imaging (Step 22-24 of 3.2.1)

\subsection{Bioluminescence imaging of Hes1 expression in dorsal telencephalon slice cultures}

1. Dissect out the uterus from pregnant mice carrying embryos at E11.5-14.5, as described above (steps 1-6 of 3.1.).

2. Wash the uterus in ice-cold PBS.

3. Dissect out embryos from the uterus.

4. Transfer the embryos into ice-cold DMEM/F12 medium.

5. Excise the brain and transfer it into a silicon rubber-coated dish with DMEM/F12, which is conditioned by a mixture of $5 \% \mathrm{CO}_{2}$ and $95 \% \mathrm{O}_{2}$ for $10-15$ min on ice.

6. Remove meninges and separate the lateral and medial regions of the telencephalon.

7. Manually slice the cortex using a microknife to make coronal slices $(100-200 \mu \mathrm{m}$ thick).

8. Transfer the cortical slices into enriched slice culture medium.

9. Put the cortical slices onto slice culture insert in a glass-based dish.

10. Add $500 \mu \mathrm{l}$ enriched slice cultured medium containing $1 \mathrm{mM}$ luciferin to the outside of slice culture insert in a glass-based dish.

11. Place the dish on the stage of inverted microscope of the bioluminescence imaging system. 
12. Maintain the slice culture at $37^{\circ} \mathrm{C}$ in $5 \% \mathrm{CO}_{2}$ and $40 \% \mathrm{O}_{2}$.

13. Collect bioluminescence from slices using an $\mathrm{x} 40$ UPlanApo objective (Olympus) and transmit the signal directly to a cooled CCD camera.

\subsection{Bioluminescence imaging of dual color luciferase reporters using the bioluminescence imaging system}

To monitor the expression of two genes simultaneously, cells carrying red-color and green-color luciferase reportes are required. These cells can be prepared by transient or stable transfection or by crossing mice that carry these reporters.

1. Prepare cells carrying the destabilized dual color luciferase reporters.

2. Plate the cells in glass-based dishes with $1 \mathrm{mM}$ D-luciferin-containing medium.

3. Place the dish on the stage of inverted microscope of the bioluminescence imaging system carrying optimized emission filters.

4. Bioluminescence from each color reporter is separated by optimized emission filters, collected by an objective lens and transmitted to cooled CCD camera.

\section{Notes}

1. We prepare 100x stock solution of bFGF (1 $\mu \mathrm{g} / \mathrm{ml}$, Invitrogen \#13256-029) dissolved in $0.1 \%$ BSA/PBS.

2. Papain and DNase are dissolved in Earle's Balanced Salt Solution (Sigma).

3. Poly-L-lysine (PLL) coating was performed as follows: Add 40 $\mu \mathrm{g} / \mathrm{ml}$ PLL solution (dissolved in water) in glass-based dishes to coat them. Incubate the dishes for $30 \mathrm{~min}$ at room temperature. Wash in PBS 3 times and dry them. 
4. We make 100x concentrated D-luciferin dissolved in PBS.

5. We put the cortical slices on culture inserts to avoid the movement of slices.

6. The maximum peak of luminescence spectrum of red-color luciferase (SLR, TOYOBO) is $630 \mathrm{~nm}$ and that of green-color luciferase (ELuc, TOYOBO) is $538 \mathrm{~nm}$.

7. We use the optimized emission filters for detect red/green color luciferase at the same time. We prepare a short pass filter $(570 \mathrm{~nm})$ for detection of the luminescence of green-color luciferase to exclude the luminescence of red-color luciferase, and a long pass filter (620nm) to detect only the luminescence of red-color luciferase.

8. Because the tip of the tail is next to the umbilical cord, it is easy to cut it accidentally. Thus, when embryos are taken out from the uterus, it is better to take out the muscle layer, which surrounds an embryo, together with the embryos.

9. Tail explants sometimes curve or move during incubation, making it difficult to focus. To avoid such movements, cut embryos at this indicated position.

10. Tips of tails are fragile and easily broken with forceps. Thus, we recommend transfering them gently using a pipette rather than using forceps. However make sure not to transfer extra medium with the tail tips to avoid alterations in the final concentration of luciferin.

11. Put the dishes on the humidified incubator, because the medium will easily dry out.

12. Choose luminescent and straight shaped tails, because curved tails will be out of focus during incubation.

13. Under these conditions, we can reproducibly monitor stable Hes7 oscillation and somite segmentation for 10-12hr. However, the tissues usually become degenerated thereafter. Using ImageJ software, we can remove cosmic rays (which make many noises), overlap the bright field images with luminescence images, and make time-lapse movies, as follows. 
Cosmic ray-induced signals were first removed [Plugins $>>$ Filters $>>$ Spike Noise Filter], and then the images were converted to 8 bit with $1,024 \times 1,024$ pixels in size by setting the maximum intensity to 255 and the minimum to 0 . Align the 8-bit images of bright field and luminescence imaging in the same region [Image $>>$ Color $>>$ Channels], and make timelapse movies.

14. In neural stem cells, the expression of Hes1 is activated by Notch signaling, which is transmitted by cell-cell interaction. Hence, cell-cell contact is important, and we usually plate cells at a high density (80-90\% confluency) to maintain the expression of Hes 1.

\section{Acknowledgement}

This work was supported by Core Research for Evolutional Science and Technology and Grantin-Aid for Scientific Research on Innovative Areas (MEXT 22123002).

\section{References}

1. Hirata, H., Yoshiura, S., Ohtsuka, T., et al. (2002) Oscillatory expression of the bHLH factor Hes1 regulated by a negative feedback loop. Science 298, 840-843

2. Masamizu, Y., Ohtsuka, T., Takashima, Y., et al. (2006) Real-time imaging of the somite segmentation clock: revelation of unstable oscillators in the individual presomitic mesoderm cells. Proc Natl Acad Sci USA 103, 1313-1318

3. Shimojo, H., Ohtsuka, T., Kageyama, R. (2008) Oscillations in Notch signaling regulate maintenance of neural progenitors. Neuron 58, 52-64

4. Kobayashi, T., Mizuno, H., Imayoshi, I., et al. (2009) The cyclic gene Hes1 contributes to diverse differentiation responses of embryonic stem cells. Genes Dev 23, 1870-1875 
5. Jarriault, S., Brou, C., Logeat, F., et al. (1995). Signalling downstream of activated mammalian Notch. Nature 377, 355-358

6. Takebayashi, K., Sasai, Y., Sakai, Y., et al. (1994) Structure, chromosomal locus, and promoter analysis of the gene encoding the mouse helix-loop-helix factor HES-1: negative autoregulation through the multiple $\mathrm{N}$ box elements. J Biol Chem 269, 5150-5156

7. Baek, J.H., Hatakeyama, J., Sakamoto, S., et al. (2006) Persistent and high levels of Hes1 expression regulate boundary formation in the developing central nervous system. Development 133, 2467-2476

8. Bessho, Y., Sakata, R., Komatsu, S., et al. (2001) Dynamic expression and essential functions of Hes7 in somite segmentation. Genes Dev 15, 2642-2647

9. Hirata, H., Bessho, Y., Kokubu, H., et al. (2004) Instability of Hes7 protein is critical for the somite segmentation clock. Nature Genet 36, 750-754

10. Takashima, Y., Ohtsuka, T., Gonzalez, A., et al. (2011) Intronic delay is essential for oscillatory expression in the segmentation clock. Proc Natl Acad Sci USA 108, 3300-3305

11. Niwa, Y., Shimojo, H., Isomura, A., et al. (2011) Different types of oscillations in Notch and Fgf signaling regulate the spatiotemporal periodicity of somitogenesis. Genes Dev 25, 1115 1120

12. Harima, Y., Takashima, Y., Ueda, Y., et al. (2012) Accelerating the tempo of the segmentation clock by reducing the number of introns in the Hes7 gene. Cell Rep 3, 1-7

13. Luker, G. D., Pica, C. M., Song, J., et al. (2003) Imaging 26S proteasome activity and inhibition in living mice. Nat Med 9, 969-973

14. Qian, X., Goderie, S.K., Shen, Q., et al. (1998) Intrinsic programs of patterned cell lineages in isolated vertebrate CNS ventricular zone cells. Development 125, 3143-3152 
15. Miyata, T., Kawaguchi, A., Okano, H., et al. (2001) Asymmetric inheritance of radial glial fibers by cortical neurons. Neuron 31, 727-741 


\section{Figure legends}

Figure 1. Schematic structures of luciferase reporters. (A) Schematic structure of pHes 1-Ub-Luc reporter. pHes1-Ub-Luc reporter consists of the Hes1 promoter, 5'UTR, Ub-Luc, Hes1 3'UTR and downstream region. (B) Schematic structure of the dual reporter system. Ubiquitinated green-color luciferase (ELuc) or red-color luciferase (SLR) was used.

Figure 2. Explant culture of the caudal part of a mouse embryo. The mouse tissue was put on a glass dish. Cutting point is indicated by arrowhead. Somites are shown by brackets, and the caudal part is PSM. Rostral is up, and caudal is down.

Figure 3. Visualization of Hes1 expression in a cultured neural stem cell. (A) Visualization of Hes 1 expression in a cultured neural stem cell carrying pHes1-Ub-Luc reporter. Luminescence was monitored by the imaging system. Each time point represents 20 -min exposure. (B) Intensity of luminescence shown in A was quantified in arbitrary units. Hes 1 expression oscillates with a period of about 2 hours in this cultured neural stem cell. 


\section{A Hes1 reporter}

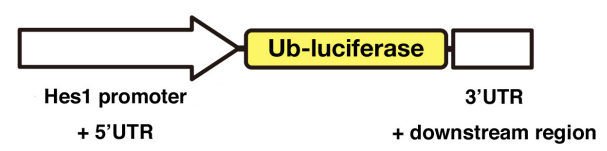

\section{B Dual reporter system}

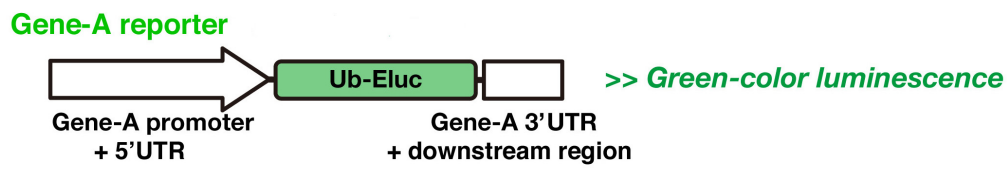

Gene-B reporter

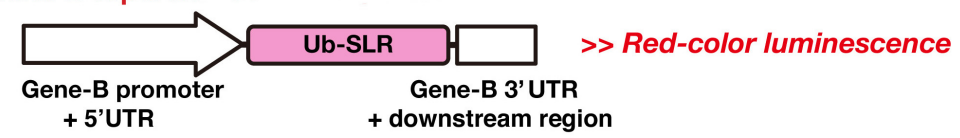

Figure 1

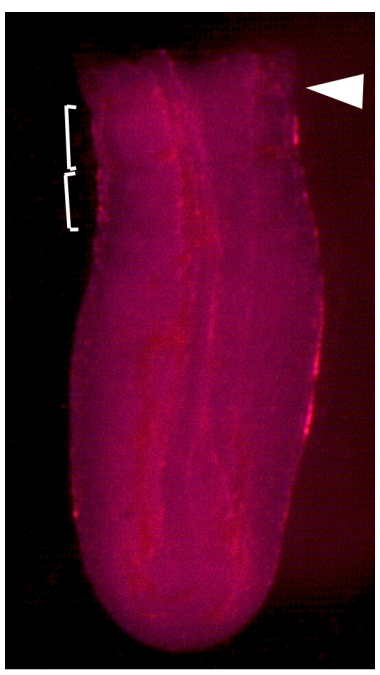

Figure 2

A

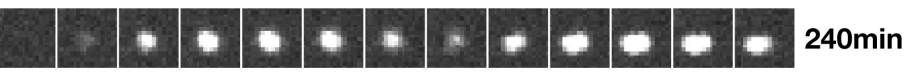

B

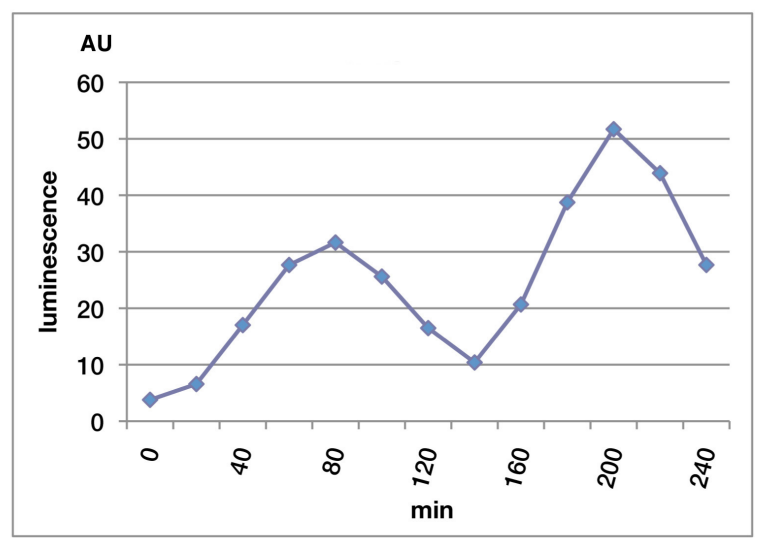

